\title{
Article \\ Mineral and Microbiological Analysis of Spices and Aromatic Herbs
}

\author{
Nicola Cicero ${ }^{1,2} \mathbb{D}^{\mathbb{D}}$, Teresa Gervasi ${ }^{1, * \mathbb{D}}$, Alessandra Durazzo ${ }^{3}\left(\mathbb{D}\right.$, Massimo Lucarini $^{3}$, Antonio Macrì ${ }^{1}$, \\ Vincenzo Nava ${ }^{1}\left(\mathbb{D}\right.$, Filippo Giarratana ${ }^{4}\left(\mathbb{D}\right.$, Roberta Tardugno ${ }^{2}$, Rossella Vadalà ${ }^{1}$ and Antonello Santini ${ }^{5, *(\mathbb{D})}$
}

1 Department of Biomedical and Dental Sciences and Morphofunctional Imaging, University of Messina, 98168 Messina, Italy; ncicero@unime.it (N.C.); macri.anto@outlook.it (A.M.); vnava@unime.it (V.N.); rosvadala@tiscali.it (R.V.)

2 Science4life, Spinoff Company, University of Messina, 98168 Messina, Italy; roberta.tardugno@gmail.com

3 CREA-Research Centre for Food and Nutrition, Via Ardeatina 546, 00178 Rome, Italy; alessandra.durazzo@crea.gov.it (A.D.); massimo.lucarini@crea.gov.it (M.L.)

4 Department of Veterinary Sciences, University of Messina, 98168 Messina, Italy; fgiarratana@unime.it

5 Department of Pharmacy, University of Napoli Federico II, Via D. Montesano 49, 80131 Napoli, Italy

* Correspondence: tgervasi@unime.it (T.G.); asantini@unina.it (A.S.)

check for

updates

Citation: Cicero, N.; Gervasi, T.;

Durazzo, A.; Lucarini, M.; Macrì, A.; Nava, V.; Giarratana, F.; Tardugno, R.; Vadalà, R.; Santini, A. Mineral and Microbiological Analysis of Spices and Aromatic Herbs. Foods 2022, 11, 548. https://doi.org/10.3390/ foods 11040548

Academic Editor: Amin

Mousavi Khaneghah

Received: 10 January 2022

Accepted: 11 February 2022

Published: 15 February 2022

Publisher's Note: MDPI stays neutral with regard to jurisdictional claims in published maps and institutional affiliations.

Copyright: (C) 2022 by the authors. Licensee MDPI, Basel, Switzerland. This article is an open access article distributed under the terms and conditions of the Creative Commons Attribution (CC BY) license (https:/ / creativecommons.org/licenses/by/ $4.0 /)$.

\begin{abstract}
Spices and aromatic herbs have always had great historical importance in human nutrition. Their use has been documented for centuries as a rich source of bioactive compounds; they have been used for their health benefits and also for flavoring or coloring food. However, despite the many health properties linked to the use of spices and aromatic herbs, these can represent biological hazards and can contain chemical substances of concern. Certainly, monitoring potential health hazards in spices and aromatic herbs includes microbiological safety and also the content of inorganic substances: both represent a key step. This research aims at monitoring the compliance of various spices and aromatic herbs from a non-European country market (namely: black cumin seeds, Iranian Tokhme Sharbati, clove buds, Shahjeera, Abbaszadeh saffron, organic fenugreek, whole black pepper, cinnamon, Abthul Ahmar (Asario), Ajwan seeds, whole coriander seeds, black sesame seeds, Sabja seeds) with the current European Union (EU) and WHO regulations, when available, regarding mineral and microbiological parameters. In particular, microbiological assays using rapid and conventional methods, and trace mineral determination by inductively coupled plasma mass spectrometry (ICP-MS) were performed. Results show the safety of the tested spices, given that the microbiological parameters were within the legal microbiological criteria set by the European Commission Regulation (EC) No. 2073/2005 and its amendment Regulation (EC) No. $1441 / 2007$. With reference to potentially toxic $\mathrm{Cd}, \mathrm{Pb}, \mathrm{As}, \mathrm{Hg}$, these were within the limits set by the European Commission Regulation (EC) No. 1881/2006 and its amendments, Regulation (EU) No. 1317/2021 and Regulation (EU) No. 1323/2021, and WHO. According to EU regulations, for Pb content, 2 samples out of 16 showed values different from the set limits.
\end{abstract}

Keywords: spices; aromatic herbs; minerals; chemical analysis; microbiological analysis

\section{Introduction}

Spices and aromatic herbs have always had great historical significance in human nutrition and in holistic approaches to health issues. In fact, they were used in ancient times not only in the food sector, i.e., to flavor and aromatize dishes, but also in the medical field, in many religious rituals, and for the preservation of food. They are documented as rich source of bioactive compounds linked to health benefits [1]. However, vegetals can be contaminated by microorganisms and can accumulate heavy metals, pesticide residues, and other potentially toxic substances from the environment depending on environmental factors, e.g., soil characteristics and absorbability, water, air, plant genotypes, and anthropogenic activities [2-9]. 
Acute or chronic poisonings may occur following heavy metal intake through food. Their bioaccumulation may lead to diverse toxic effects on a variety of body tissues and organs. Heavy metals disrupt cellular events including growth, proliferation, differentiation, damage-repairing processes, and apoptosis [10].

Thus, spices and aromatic herbs can be biological and chemical threats when used as food ingredients or for medicinal uses [11-13]. The conservation of spices and aromatic herbs is carried out in most cases via the dehydration process, through physical processes such as heat and/or pressure. This conservation methodology is minimally invasive and aims to inhibit metabolic activities, and therefore the proliferation of microorganisms by subtracting the free and bound water within the food. Dehydrated foods are characterized by a low moisture content $(<14 \%)$, corresponding to a low value of free water with water activity $\left(A_{W}\right)$ that is less than 0.75 . Compared to the native product, the reduction in $A_{W}$ values therefore represent one of the main parameters of ensuring the inhibition of the growth of organisms, consequently giving stability to the food from both a microbiological, enzymatic, and chemical point of view. Foodstuffs of vegetable origin such as cereals and derivatives, seeds, dried fruit, coffee, cocoa, herbs, and spices are easily attacked by molds, which in particular conditions of temperature and humidity, can produce secondary potentially dangerous metabolites such as mycotoxins [14-18].

Although spices and aromatic herbs on the market are dehydrated products and therefore have stable chemical and physical characteristics, these foods are generally subjected to drying processes at room temperature in their places of origin, which generally are developing or tropical countries where the production technologies used are not always capable of guaranteeing the implementation of good hygiene and safety practices, thus becoming the main cause of contamination $[19,20]$. Nevertheless, even with low water activity levels, some microorganisms, including pathogenic and toxigenic ones, are able to survive and may proliferate when vegetal matrices are added to foods.

Considering the crucial role of safety and quality in food production, in Europe, systems to detect and neutralize contaminants in herbs and spices have been developed within the project "Securing the spices and herbs commodity chains in Europe against deliberate, accidental, or natural biological and chemical contamination" (SPICED). The EU market is one of the main world markets for spices and herbs, and the problem linked to microbiological and toxicological hazards can pose a serious risk for the consumer, as spices and herbs could potentially contaminate a wide range of products due to their widespread use. Europe is one of the most important regions in the world with reference to the importation of herbs and spices, accounting for about one quarter of the world's total imports of herbs and spices [21,22].

Different microorganisms could be potentially harmful in herbs as well as in different food matrices, i.e., Staphylococcus aureus, Salmonella spp., Escherichia coli, Listeria monocytogenes, aflatoxin-producing fungi (i.e., Aspergillus spp.), Clostridium perfringens, and Bacillus cereus $[16,23-25]$.

Taking into account these possible threats to health, the problem of spice and herb product consumption has global significance.

There are many studies on microflora in agricultural products, but only a small part of them address spices and officinal and aromatic herbs, which are instead increasingly present in our daily diet, thus also acquiring an increasingly important economic role.

On the other hand, monitoring the presence of metals in spices and herbs represents a key step [25-27].

Metals have important biological functions and activities, but inorganic elements can become toxic when their intake exceeds the accepted and maximum allowed levels as suggested by the European Commission and the European Food Safety Authority (EFSA) [28-36].

Considering that Europe is among the main importer of spices from extra EU countries [21,22], monitoring the contaminant levels in spices and herbs from these countries may provide relevant toxicological data on spices and aromatic herbs commonly present in the 
European Union (EU) market, improving the accuracy of dietary risk exposure/assessment, and thus enhancing the feasibility of epidemiological studies. In this context, the present study aims to monitor the safety of some spices and herbs from foreign countries by evaluating: (i) microbiological contamination, and (ii) trace mineral element content.

\section{Materials and Methods}

\subsection{Samples}

Spices and aromatic herbs of different species, and of different countries of origin, were acquired in an international market in Saudi Arabia. The spices and herbs that were the object of this study are among the main ones imported into Europe and distributed in European countries. In this context, the end points of the present study were to check conformity with existing EU regulations, and the content of trace metal elements.

Thirteen samples of spices and aromatic herbs (labelled from A to O), packed in sealed plastic bags, were subjected to microbiological and chemical analysis. Table 1 reports the description of the samples according to their scientific name, vulgaris name, and country of origin. All microbial and chemical analyses have been carried out in triplicate.

Table 1. Analyzed herbs and spices and their origin.

\begin{tabular}{llll}
\hline Label & Origin & Common Name & Scientific Name \\
\hline Black Cumin Seeds (A) & India & Black cumin & Nigella sativa \\
Iranian Tokhme Sharbati (B) & Iran & Chia seeds & Salvia hispanica \\
Clove Buds (C) & Indonesia & Clove buds & Syzygium aromaticum \\
Shahjeera (D) & India & Caraway & Carum carvi \\
Abbaszadeh Saffron (E) & Iran & Saffron & Crocus sativus \\
Organic Fenugreek (F) & India & Fenugreek & Trigonella foenum-graecum \\
Whole Black Pepper (G) & Vietnam & Black pepper & Piper nigrum \\
Cinnamon (H) & Indonesia & Cinnamon & Cinnamomum verum \\
Abthul Ahmar (Asario) (I) & India & Cress Sprouting Seeds & Lapidium sativum \\
Ajwan Seeds (L) & India & Thymol seeds & Trachyspermum ammi \\
Whole Coriander Seeds (M) & India & Coriander & Coriandrum sativum \\
Black Sesame Seeds (N) & India & Black sesame seeds & Sesamum indicum \\
Sabja Seeds (O) & India & Chia seeds & Salvia hispanica \\
\hline
\end{tabular}

\subsection{Microbiological Methodology}

The counts of total mesophilic bacteria, fungi (yeasts and molds), total coliforms, Escherichia coli, Enterobacteria, lactic bacteria, Staphylococcus aureus, Salmonella spp., Listeria monocytogenes, Bacillus cereus, and sulphite-reducing clostrides were performed. All media were supplied by ThermoFisher Scientific, Oxoid Ltd., Basingstoke, UK. The samples were homogenized with PBS ( $\mathrm{pH}=7.4$ ) by mixing vigorously. Then serial dilutions in PBS $(\mathrm{pH}=7.4)$ were performed, and the samples were spread aseptically over the media plates. For mesophilic counts, samples were spread over plate count agar (PCA) and incubated at $30{ }^{\circ} \mathrm{C}$ for $72 \mathrm{~h}$. Malt extract agar with $10 \%$ lactic acid (MEA) was utilized to verify the counts of yeasts and molds by incubating at room temperature for 3 days. Lactic acid microorganisms and Enterobacteriacee were investigated using De Man, Rogosa, and Sharpe agar and Violet Red Bile Glucose Agar (MRSA and VRBG) using an incubation period of $72 \mathrm{~h}$ at $30^{\circ} \mathrm{C}$ and $48 \mathrm{~h}$ at $37^{\circ} \mathrm{C}$, respectively. The determination of B. cereus was carried out by using Bacillus Cereus Agar Base (PEMBA) after an incubation for $24 \mathrm{~h}$ at $37^{\circ} \mathrm{C}$. Coliforms and E. coli were analyzed using the standard membrane filter technique using Chromogenic Coliform Agar (CCA) and Tryptone Bile X-Gluc agar (TBX) using an incubation period of $24 \mathrm{~h}$ at $37^{\circ} \mathrm{C}$ and of $18 / 24 \mathrm{~h}$ at $44{ }^{\circ} \mathrm{C}$. S. aureus contamination was detected using the standard membrane filter technique using Baird-Parker Agar (BPA), at $37^{\circ} \mathrm{C}$ for $48 \mathrm{~h}$. The standard membrane filter technique using Sulfite Polymyxin Sulfadizine agar (SPS) was used for the determination of sulphite-reducing clostrides after an incubation at $37^{\circ} \mathrm{C}$ for $48 \mathrm{~h}$. The determination of L. monocytogenes was carried out according to ISO standard 11290. Briefly, after a two-stage enrichment process, the first 
in half Fraser broth for $24 \mathrm{~h}$ and then in Fraser broth, the enriched broths were plated on Oxford and BD PALCAM Lysteria agars.

The presence of Salmonella spp. was investigated according to ISO standard 6579, which includes: pre-enrichment in a non-selective liquid medium (buffered pepton water), followed by selective enrichment (Rappaport-Vessiliadis Soy Broth; ThermoFisher Scientific, Oxoid Ltd., Basingstoke, UK), and then isolation on a selective medium (Hektoen; ThermoFisher Scientific, Oxoid Ltd., Basingstoke, UK). All microbial analyses were carried out in triplicate.

\subsection{Mineral Analysis}

\subsubsection{Sample Preparation}

A total of $0.25 \mathrm{~g}$ dry weight $(\mathrm{dw})$ of each sample was milled in a Teflon mortar and digested with $7 \mathrm{~mL}$ of $65 \%(v / v) \mathrm{HNO}_{3}$, and $1 \mathrm{~mL}$ of $30 \%(v / v) \mathrm{H}_{2} \mathrm{O}_{2}$ (J.T. Baker, Mallinckrodt Baker, Milan, Italy). A total of $1 \mathrm{~mL}$ of $0.8 \mathrm{mg} \mathrm{L}^{-1}$ Re (Fluka, Milan, Italy) was added as an internal standard. Mineralization was performed in an Ethos 1 digestor (Milestone, Bergamo, Italy) at $1000 \mathrm{~W}$ and $180^{\circ} \mathrm{C}$; this temperature was reached in $10 \mathrm{~min}$ and held for another $10 \mathrm{~min}$. The digested samples, cooled at room temperature, were diluted with ultrapure deionized water obtained (J.T. Baker, Mallinckrodt Baker, Milan, Italy) and stored at $4{ }^{\circ} \mathrm{C}$.

\subsubsection{ICP-MS Analysis}

Minerals were determined by the same procedure utilized for the determination of potentially toxic inorganic species in vegetables as reported in a previous work [16]. An Agilent 7500CX ICP-MS spectrometer (Agilent, Santa Clara, CA, USA) equipped with an Octapole Reaction System (ORS) reaction/collision cell, and with an ASX 500 auto sampler, was used for analyzing the digested samples. The system was pressurized with $99.9 \%$ pure helium (Rivoira S.p.A., Milan, Italy).

The operating conditions of the ICP-MS were as follows: RF power, $1550 \mathrm{~W}$; plasma gas flow rate, $15 \mathrm{~L} \mathrm{~min}^{-1}$; auxiliary gas flow rate, $0.9 \mathrm{~L} \mathrm{~min}^{-1}$; carrier gas flow rate, $1.1 \mathrm{~L} \mathrm{~min}^{-1}$; sample introduction flow rate, $1 \mathrm{~mL} \mathrm{~min}^{-1}$; sample depth, $9 \mathrm{~mm}$; spray chamber temperature $2{ }^{\circ} \mathrm{C}$; vacuum, $<1.5 \times 10^{-7} \mathrm{~Pa}$; interface pressure, $5.3 \times 10^{-2} \mathrm{~Pa}$.

${ }^{7} \mathrm{Li},{ }^{59} \mathrm{Co},{ }^{80} \mathrm{Y}$, and ${ }^{205} \mathrm{Tl}$ solutions (Agilent, Santa Clara, CA, USA) at a concentration of $10 \mu \mathrm{g} \mathrm{L}^{-1}$ were used for tuning the instrument.

${ }^{63} \mathrm{Cu},{ }^{60} \mathrm{Ni},{ }^{75} \mathrm{As},{ }^{51} \mathrm{~V},{ }^{52} \mathrm{Cr}$, and ${ }^{208,207,206} \mathrm{~Pb}$ (Fluka, Milan, Italy), and ${ }^{114} \mathrm{Cd}$ and ${ }^{202} \mathrm{Hg}$ isotopes (Merck, Darmstadt, Germany) were selected to optimize the sensitivity, and to minimize matrix interference.

A solution of ${ }^{115} \mathrm{In},{ }^{45} \mathrm{Sc},{ }^{103} \mathrm{Rh}$, and ${ }^{209} \mathrm{Bi}$ at a concentration of $10 \mu \mathrm{g} \mathrm{L}{ }^{-1}$ was used as an online internal standard to correct any instrumental drifts and matrix effects.

Quantitative determinations were performed using the external standard method. The calibration used a multi-standard solution of $\mathrm{Cr}, \mathrm{V}, \mathrm{Cu}, \mathrm{Cd}, \mathrm{Pb}$, and $\mathrm{Ni}$ at different concentrations ranging from 0.5 to $2000 \mu \mathrm{g} \mathrm{L}-1$.

$\mathrm{Hg}$ was analyzed separately following a previously described procedure [5]. All analyses were carried out in triplicate.

\subsubsection{Statistical Analysis}

All mineral data are reported as the mean and standard deviation of three independent determinations. With regards to the selected heavy metals $(\mathrm{Cd}, \mathrm{Pb}, \mathrm{As}$, and $\mathrm{Hg})$, their contents were statistically compared with the reference limit values established by the European Commission and WHO by means of Student's $t$ test. The statistical analysis has been performed using the Past software (Version 4.09) [37].

\section{Results and Discussion}

The safety aspects that were explored throughout the study were: (i) microbiological aspects; and (ii) mineral profiles. 


\subsection{Microbiological Analysis}

The microbiological analysis of 13 dried spices, labelled with letters from $A$ to $\mathrm{O}$, is reported in Table 2 .

Table 2. Bacterial contamination $\left(\mathrm{CFU} \mathrm{g}^{-1}\right)$ of dried spices from India (a) and Iran, Indonesia, and Vietnam $(\mathbf{b})$.

\begin{tabular}{|c|c|c|c|c|c|c|c|c|c|}
\hline \multicolumn{10}{|c|}{ (a) } \\
\hline Microorganisms & A & D & $\mathbf{F}$ & I & $\mathbf{L}$ & $\mathbf{M}$ & $\mathbf{N}$ & $\mathrm{O}$ & $\begin{array}{l}\text { Reference } \\
\text { Values }^{\text {a }}\end{array}$ \\
\hline $\begin{array}{l}\text { Mesophilic } \\
\text { Bacteria }\end{array}$ & $2.1 \times 10^{4}$ & $1.7 \times 10^{6 *}$ & $3.7 \times 10^{2}$ & 30 & $1.6 \times 10^{3}$ & $2.4 \times 10^{2}$ & $3.2 \times 10^{2}$ & $8.5 \times 10^{4}$ & $<5 \times 10^{5}$ \\
\hline Molds & $1.2 \times 10^{2}$ & $2.3 \times 10^{2}$ & $1 \cdot 10$ & ND & ND & ND & $1.2 \times 10^{2}$ & ND & $<1 \times 10^{3}$ \\
\hline Yeasts & 30 & $7.1 \times 10^{2}$ & ND & ND & ND & ND & ND & ND & $<1 \times 10^{3}$ \\
\hline Coliforms & $7 \times 10^{3 *}$ & $2.4 \times 10^{4 *}$ & ND & ND & ND & ND & $6.2 \times 10^{3}$ & ND & $<1 \times 10^{3}$ \\
\hline Escherichia coli & ND & ND & ND & ND & ND & ND & ND & ND & $<10$ \\
\hline $\begin{array}{l}\text { Sulfite-Reducing } \\
\text { Clostridia }\end{array}$ & ND & ND & ND & ND & ND & ND & ND & ND & $<1 \times 10^{2}$ \\
\hline Clostridial Spores & ND & ND & ND & ND & ND & ND & ND & ND & $<1 \times 10^{2}$ \\
\hline $\begin{array}{l}\text { Staphilococcus } \\
\text { aureus }\end{array}$ & ND & ND & ND & ND & ND & ND & ND & ND & $<1 \times 10^{2}$ \\
\hline Salmonella spp. ${ }^{\mathrm{b}}$ & ND & ND & ND & ND & ND & ND & ND & ND & 0 \\
\hline $\begin{array}{l}\text { Listeria } \\
\text { monocytogenes }\end{array}$ & ND & ND & ND & ND & ND & ND & ND & ND & $<10^{2}$ \\
\hline Bacillus cereus & ND & ND & ND & ND & ND & ND & $2 \times 10$ & ND & $<1 \times 10^{3}$ \\
\hline Enterobatteriacee & $1.4 \times 10^{4 *}$ & $9 \times 10^{4 *}$ & ND & ND & ND & ND & $4.4 \times 10^{3 *}$ & ND & $<10$ \\
\hline Lactic Bacteria & ND & ND & ND & ND & ND & ND & ND & ND & $<1 \times 10^{5}$ \\
\hline \multicolumn{10}{|c|}{ (b) } \\
\hline Microorg & sms & B & $\mathrm{C}$ & $\mathbf{E}$ & G & $\mathbf{H}$ & I & \multicolumn{2}{|c|}{ Reference Values a } \\
\hline Mesofilic & teria & $6 \times 10^{4}$ & $2.2 \times 10^{2}$ & $2.5 \times 10^{2}$ & $9.3 \times 10^{4}$ & $1.1 \times 10^{2}$ & 30 & \multicolumn{2}{|c|}{$<5 \times 10^{5}$} \\
\hline Mol & & $2.5 \times 10^{2}$ & ND & ND & ND & ND & ND & \multicolumn{2}{|c|}{$<1 \times 10^{3}$} \\
\hline Yeas & & ND & ND & ND & ND & ND & ND & \multicolumn{2}{|c|}{$<1 \times 10^{3}$} \\
\hline Colifo & & $1.6 \times 10^{3 *}$ & $1.7 \times 10^{2}$ & ND & $4 \cdot 10$ & ND & ND & \multicolumn{2}{|c|}{$<1 \times 10^{3}$} \\
\hline Escherich & coli & ND & ND & ND & ND & ND & ND & \multicolumn{2}{|c|}{$<1 \times 10$} \\
\hline $\begin{array}{l}\text { Sulfite-Re } \\
\text { Clostr }\end{array}$ & cing & ND & ND & ND & ND & ND & ND & \multicolumn{2}{|c|}{$<1 \times 10^{2}$} \\
\hline Clostridia & ores & ND & ND & ND & ND & ND & ND & \multicolumn{2}{|c|}{$<1 \times 10^{2}$} \\
\hline Staphilococc & aureus & ND & ND & ND & ND & ND & ND & \multicolumn{2}{|c|}{$<1 \times 10^{2}$} \\
\hline Salmonell & p. ${ }^{b}$ & ND & ND & ND & ND & ND & ND & \multicolumn{2}{|c|}{0} \\
\hline Listeria mon & togenes & ND & ND & ND & ND & ND & ND & \multicolumn{2}{|c|}{$1 \times 10^{2}$} \\
\hline Bacillus & & ND & ND & ND & ND & ND & ND & \multicolumn{2}{|c|}{$<1 \times 10^{3}$} \\
\hline Enterobat & acee & $6.6 \times 10^{2 *}$ & $2 \times 10^{*}$ & ND & ND & ND & ND & \multicolumn{2}{|c|}{$<10$} \\
\hline Lactic $B$ & & ND & ND & ND & ND & ND & ND & \multicolumn{2}{|c|}{$<1 \times 10^{5}$} \\
\hline
\end{tabular}

${ }^{a}$ Reference values for microbiological safety and quality were based on regulations from the European Union and other international guidelines $[8,38,39] .{ }^{\mathrm{b}} \mathrm{CFU} 25 \mathrm{~g}^{-1}$. * Values are higher than the reference values. ND, not determined. A, black cumin seeds; B, Iranian Tokhme Sharbati; C, clove buds; D, Shahjeera; E, Abbaszadeh saffron; F, organic fenugreek; G, whole black pepper; H, cinnamon; I, Abthul Ahmar (Asario); L, Ajwan seeds; M, whole coriander seeds; N, black sesame seeds; O, Sabja seeds.

According to the Commission Regulation (EC) No. 2073/2005 and its amendment Regulation (EC) No. 1441/2007, which sets legal microbiological criteria for several food products, these spices could be defined safe products $[8,38,39]$.

With regards to the indicator microorganisms, which are used to provide simple, reliable, and rapid information about processing failures, post-processing contamination from the environment, the general level of hygiene, and the presence or absence of foodborne pathogens to monitor the chain of food production, low bacterial loads were detected in saffron, fenugreek, black pepper, cinnamon, cress sprouting seed, thymol seed, coriander, and chia seed [40].

The total coliform and Enterobacteriaceae amounts in black cumin were $7 \times 10^{3} \mathrm{CFU} \mathrm{g}^{-1}$ and $1.4 \times 10^{4} \mathrm{CFU} \mathrm{g}^{-1}$, respectively, whereas in Iranian chia seeds it was $1.6 \times 10^{3} \mathrm{CFU} \mathrm{g}^{-1}$ and $6.6 \times 10^{2} \mathrm{CFU} \mathrm{g}^{-1}$, respectively. Caraway presented a mesophilic count of $1.7 \times 10^{6} \mathrm{CFU} \mathrm{g}^{-1}$, and an amount of total coliform and Enterobacteriaceae of $2.4 \times 10^{4} \mathrm{CFU} \mathrm{g}-1$ and $9 \times 10^{4} \mathrm{CFU} \mathrm{g}{ }^{-1}$, respectively. Moreover, Enterobacteriaceae were also detected in clove buds $\left(2 \times 10 \mathrm{CFU} \mathrm{g}^{-1}\right)$ and black sesame seeds $\left(4.4 \times 10^{3} \mathrm{CFU} \mathrm{g}^{-1}\right)$. 
These contaminations may be related to the environment, inadequate hygienic handling, unsanitary conditions, and others, and occurs in samples purchased in street markets. Indeed, the adoption of good hygiene practices in all the involved steps from land growing, harvesting, and processing can be useful to reduce the health risks of spice consumption.

\subsection{Mineral Analysis}

\subsubsection{Method Validation}

The method was validated according to Eurachem criteria [41]. Commercial standards were used for the evaluation of method linearity, limits of detection (LODs), limits of quantification (LOQs), accuracy, repeatability, and intermediate precision. LODs and LOQs were calculated as $3.3 \sigma / \mathrm{S}$ and $10 \sigma / \mathrm{S}$, respectively, where $\sigma$ is the standard deviation of six blanks and $\mathrm{S}$ is the slope of the relative calibration curve. A good linearity was obtained for all elements investigated with R2 values ranging from 0.9992 (for $\mathrm{Cu}$ and Se) to 0.9999 (for V). The limits of detection (LODs) ranged from 0.001 to $0.051 \mu \mathrm{g} \mathrm{kg}^{-1}$, and the limits of quantification (LOQs) ranged from 0.003 to $0.168 \mu \mathrm{g} \mathrm{kg}^{-1}$. The lowest average recovery was observed for mercury with $92.93 \%$, while the highest was obtained for strontium with $103.03 \%$. Accuracy was assessed by evaluating six determinations on certified reference materials (NIST1570A spinach leaves) and was reported as the percent recovery between the value found with the calibration curve and the true value reported in the certified reference materials. If the element was not certified in the reference materials, the matrix was spiked with the known amount of analyte, and was analyzed following the procedures discussed previously. Based on these results, the analytical characteristic (linearity, sensitivity, and accuracy) can be considered to be satisfactory for the purposes of the analysis (Table 3 ).

Table 3. Analytical parameters for method validation.

\begin{tabular}{|c|c|c|c|c|c|c|}
\hline Element & $\begin{array}{c}\text { LOD } \\
\left(\mu \mathrm{kg}^{-1}\right)\end{array}$ & $\begin{array}{c}\mathrm{LOQ} \\
\left(\mu \mathrm{gg}^{-1}\right)\end{array}$ & $\mathbf{R}^{2}$ & $\begin{array}{c}\text { Experimental } \\
\text { Value } \\
\left(\mathrm{mg} \mathrm{kg}^{-1}\right)\end{array}$ & $\begin{array}{l}\text { Expected } \\
\text { Value } \\
\left(\mathrm{mg} \mathrm{kg}^{-1}\right)\end{array}$ & $\begin{array}{c}\text { Recovery } \\
(\%)\end{array}$ \\
\hline $\mathrm{Be}$ & 0.001 & 0.003 & 0.9998 & 1.90 * & $2.00 *$ & 95.00 \\
\hline Sn & 0.005 & 0.017 & 0.9997 & $1.97 *$ & $2.00 *$ & 98.50 \\
\hline $\mathrm{Al}$ & 0.051 & 0.168 & 0.9995 & 308.55 & 310.00 & 99.53 \\
\hline V & 0.003 & 0.010 & 0.9999 & 0.575 & 0.568 & 101.23 \\
\hline $\mathrm{Cr}$ & 0.003 & 0.010 & 0.9997 & 1.98 & 2.00 * & 99.00 \\
\hline Mn & 0.015 & 0.050 & 0.9996 & 75.3 & 76.0 & 99.08 \\
\hline $\mathrm{Fe}$ & 0.035 & 0.116 & 0.9994 & 2.02 & 2.00 * & 101.00 \\
\hline Co & 0.002 & 0.007 & 0.9995 & 0.389 & 0.393 & 98.98 \\
\hline $\mathrm{Ni}$ & 0.002 & 0.007 & 0.9997 & 2.115 & 2.142 & 98.74 \\
\hline $\mathrm{Cu}$ & 0.025 & 0.083 & 0.9992 & 11.98 & 12.22 & 98.04 \\
\hline $\mathrm{Zn}$ & 0.032 & 0.106 & 0.9993 & 83.0 & 82.3 & 100.85 \\
\hline As & 0.001 & 0.003 & 0.9998 & 0.070 & 0.068 & 102.94 \\
\hline Se & 0.012 & 0.040 & 0.9992 & 0.1112 & 0.1152 & 96.53 \\
\hline $\mathrm{Sr}$ & 0.09 & 0.030 & 0.9996 & 57.22 & 55.54 & 103.03 \\
\hline $\mathrm{Cd}$ & 0.001 & 0.003 & 0.9997 & 2.831 & 2.876 & 98.44 \\
\hline $\mathrm{Sb}$ & 0.002 & 0.007 & 0.9996 & 1.96 * & 2.00 * & 98.00 \\
\hline $\mathrm{Pb}$ & 0.002 & 0.007 & 0.9996 & 0.19 & 0.20 & 95.00 \\
\hline $\mathrm{Hg}$ & 0.001 & 0.003 & 0.9998 & 0.0276 & 0.0297 & 92.93 \\
\hline
\end{tabular}

${ }^{*}$ Not present in the certified matrix. Element added for method validation.

\subsubsection{Mineral Contents}

The content of inorganic elements in the samples, labelled with letters from A to $\mathrm{O}$, are shown in Table 4 and reported as average values and standard deviations. 
Table 4. Trace elements (a), potentially non-toxic elements (b), and potentially toxic elements (c) present in dried spices. The contents are expressed as the mean value $\left(\mathrm{mg} \mathrm{kg}^{-1}\right)$ and standard deviation.

\begin{tabular}{|c|c|c|c|c|c|c|}
\hline \multicolumn{7}{|c|}{ (a) } \\
\hline & $\mathrm{Cr}$ & $\mathrm{Fe}$ & Mn & $\mathrm{Cu}$ & Se & $\mathrm{Zn}$ \\
\hline A & $0.04 \pm 0.001$ & $268.850 \pm 12.750$ & $15.87 \pm 0.558$ & $11.089 \pm 0.380$ & $0.072 \pm 0.001$ & $44.060 \pm 1.946$ \\
\hline B & $0.059 \pm 0.000$ & $101.221 \pm 7.653$ & $11.925 \pm 0.045$ & $10.014 \pm 0.059$ & $0.244 \pm 0.002$ & $31.539 \pm 0.066$ \\
\hline $\mathrm{C}$ & $0.119 \pm 0.006$ & $136.998 \pm 9.447$ & $627.263 \pm 28.417$ & $2.599 \pm 0.104$ & $0.011 \pm 0.001$ & $5.798 \pm 0.332$ \\
\hline $\mathrm{D}$ & $0.193 \pm 0.002$ & $303.000 \pm 10.300$ & $14.839 \pm 0.520$ & $8.366 \pm 0.023$ & $0.254 \pm 0.008$ & $20.982 \pm 0.100$ \\
\hline $\mathrm{E}$ & $0.314 \pm 0.015$ & $251.211 \pm 6.452$ & $13.964 \pm 0.538$ & $6.837 \pm 0.379$ & $0.028 \pm 0.001$ & $30.157 \pm 1.764$ \\
\hline $\mathrm{F}$ & $0.021 \pm 0.000$ & $314.231 \pm 6.921$ & $9.114 \pm 0.026$ & $8.631 \pm 0.095$ & $0.052 \pm 0.008$ & $30.013 \pm 0.267$ \\
\hline G & $0.096 \pm 0.003$ & $13.736 \pm 1.326$ & $95.802 \pm 0.455$ & $5.879 \pm 0.054$ & $0.008 \pm 0.001$ & $6.085 \pm 0.082$ \\
\hline $\mathrm{H}$ & $2.357 \pm 0.029$ & $10.586 \pm 2.531$ & $85.896 \pm 0.936$ & $2.376 \pm 0.080$ & $0.003 \pm 0.001$ & $15.652 \pm 0.013$ \\
\hline $\mathrm{I}$ & $0.088 \pm 0.044$ & $86.554 \pm 4.586$ & $16.747 \pm 1.330$ & $2.871 \pm 0.052$ & $0.287 \pm 0.003$ & $35.357 \pm 0.187$ \\
\hline $\mathrm{L}$ & $1.113 \pm 0.010$ & $289.667 \pm 10.441$ & $19.505 \pm 0.024$ & $6.874 \pm 0.023$ & $0.254 \pm 0.003$ & $48.049 \pm 0.035$ \\
\hline M & $0.129 \pm 0.007$ & $192.395 \pm 13.521$ & $16.289 \pm 0.607$ & $10.266 \pm 0.085$ & $0.505 \pm 0.009$ & $26.638 \pm 309$ \\
\hline $\mathrm{N}$ & $0.152 \pm 0.001$ & $124.569 \pm 5.114$ & $10.536 \pm 0.168$ & $11.804 \pm 0.037$ & $0.485 \pm 0.006$ & $36.698 \pm 0.148$ \\
\hline $\mathrm{O}$ & $0.109 \pm 4.813$ & $95.142 \pm 4.236$ & $103.145 \pm 4.813$ & $6.321 \pm 0.284$ & $0.009 \pm 0.001$ & $6.843 \pm 0.263$ \\
\hline
\end{tabular}

(b)

\begin{tabular}{|c|c|c|c|c|c|c|c|}
\hline & $\mathrm{Sr}$ & $\mathrm{Ni}$ & Sn & $\mathbf{V}$ & Co & $\mathrm{Sb}$ & Be \\
\hline A & $17.404 \pm 0.716$ & $3.053 \pm 0.123$ & $0.052 \pm 0.001$ & $0.028 \pm 0.001$ & $0.052 \pm 0.001$ & $0.001 \pm 0.000$ & $<\mathrm{LOD}$ \\
\hline B & $62.373 \pm 0.023$ & $0.416 \pm 0.004$ & $0.510 \pm 0.019$ & $0.084 \pm 0.001$ & $0.271 \pm 0.001$ & $0.004 \pm 0.002$ & $<\mathrm{LOD}$ \\
\hline $\mathrm{C}$ & $36.705 \pm 1.657$ & $0.753 \pm 0.034$ & $0.108 \pm 0.038$ & $0.040 \pm 0.001$ & $0.048 \pm 0.002$ & $0.008 \pm 0.008$ & $<\mathrm{LOD}$ \\
\hline $\mathrm{D}$ & $95.917 \pm 0.197$ & $0.892 \pm 0.010$ & $0.617 \pm 0.017$ & $0.138 \pm 0.001$ & $0.111 \pm 0.001$ & $0.006 \pm 0.001$ & $<\mathrm{LOD}$ \\
\hline $\mathrm{E}$ & $7.479 \pm 0.302$ & $1.124 \pm 0.069$ & $0.126 \pm 0.073$ & $0.303 \pm 0.013$ & $0.094 \pm 0.005$ & $0.010 \pm 0.001$ & $<\mathrm{LOD}$ \\
\hline $\mathrm{F}$ & $4.299 \pm 0.021$ & $0.639 \pm 0.010$ & $<\mathrm{LOD}$ & $0.021 \pm 0.001$ & $0.227 \pm 0.001$ & $0.003 \pm 0.000$ & $<\mathrm{LOD}$ \\
\hline $\mathrm{G}$ & $8.667 \pm 0.047$ & $1.194 \pm 0.009$ & $1.174 \pm 0.011$ & $0.066 \pm 0.001$ & $0.020 \pm 0.001$ & $0.003 \pm 0.002$ & $<\mathrm{LOD}$ \\
\hline $\mathrm{H}$ & $50.586 \pm 0.127$ & $2.766 \pm 0.012$ & $1.054 \pm 0.028$ & $0.052 \pm 0.001$ & $0.048 \pm 0.001$ & $0.013 \pm 0.000$ & $<\mathrm{LOD}$ \\
\hline I & $9.414 \pm 0.929$ & $0.732 \pm 0.045$ & $0.804 \pm 0.007$ & $0.024 \pm 0.001$ & $0.053 \pm 0.001$ & $0.005 \pm 0.001$ & $<\mathrm{LOD}$ \\
\hline $\mathrm{L}$ & $89.403 \pm 1.563$ & $2.087 \pm 0.021$ & $0.813 \pm 0.006$ & $1.415 \pm 0.013$ & $0.288 \pm 0.004$ & $0.016 \pm 0.000$ & $<\mathrm{LOD}$ \\
\hline M & $15.222 \pm 0.219$ & $1.134 \pm 0.011$ & $2.392 \pm 0.039$ & $0.049 \pm 0.006$ & $0.132 \pm 0.001$ & $0.022 \pm 0.016$ & $<\mathrm{LOD}$ \\
\hline $\mathrm{N}$ & $55.034 \pm 0.490$ & $0.928 \pm 0.013$ & $0.735 \pm 0.013$ & $0.062 \pm 0.001$ & $0.145 \pm 0.002$ & $0.003 \pm 0.003$ & $<\mathrm{LOD}$ \\
\hline $\mathrm{O}$ & $9.547 \pm 0.287$ & $1.293 \pm 0.061$ & $1.439 \pm 0.129$ & $0.073 \pm 0.001$ & $0.022 \pm 0.001$ & $0.028 \pm 0.034$ & $<\mathrm{LOD}$ \\
\hline
\end{tabular}

(c)

\begin{tabular}{cccccc}
\hline & $\mathbf{C d}$ & $\mathbf{P b}$ & As & Hg & Al \\
\hline A & $0.026 \pm 0.001$ & $0.034 \pm 0.001$ & $0.024 \pm 0.002$ & $0.01 \pm 0.001$ & $19.963 \pm 0.742$ \\
$\mathrm{~B}$ & $0.010 \pm 0.000$ & $0.023 \pm 0.000$ & $0.015 \pm 0.000$ & $0.01 \pm 0.000$ & $32.355 \pm 0.008$ \\
$\mathrm{C}$ & $0.014 \pm 0.000$ & $0.034 \pm 0.001$ & $0.005 \pm 0.000$ & $0.003 \pm 0.001$ & $20.532 \pm 1.097$ \\
$\mathrm{D}$ & $0.012 \pm 0.001$ & $0.043 \pm 0.000$ & $0.078 \pm 0.001$ & $0.003 \pm 0.001$ & $87.507 \pm 0.269$ \\
\hline $\mathrm{E}$ & $0.017 \pm 0.001$ & $0.096 \pm 0.005$ & $0.086 \pm 0.002$ & $0.003 \pm 0.001$ & $145.216 \pm 6.281$ \\
$\mathrm{~F}$ & $0.011 \pm 0.000$ & $0.010 \pm 0.001$ & $0.003 \pm 0.000$ & $0.002 \pm 0.001$ & $7.112 \pm 0.108$ \\
$\mathrm{G}$ & $0.003 \pm 0.000$ & $0.008 \pm 0.000$ & $0.002 \pm 0.000$ & $0.003 \pm 0.001$ & $43.064 \pm 0.253$ \\
$\mathrm{H}$ & $0.071 \pm 0.001$ & $0.023 \pm 0.000$ & $0.005 \pm 0.000$ & $0.002 \pm 0.001$ & $27.168 \pm 0.547$ \\
$\mathrm{I}$ & $0.079 \pm 0.001$ & $0.012 \pm 0.001$ & $0.009 \pm 0.000$ & $0.002 \pm 0.001$ & $8.863 \pm 0.390$ \\
$\mathrm{~L}$ & $0.065 \pm 0.000$ & $0.544 \pm 0.003$ & $0.339 \pm 0.006$ & $0.004 \pm 0.001$ & $930.198 \pm 5.269$ \\
$\mathrm{M}$ & $0.034 \pm 0.002$ & $0.026 \pm 0.002$ & $0.009 \pm 0.002$ & $0.004 \pm 0.002$ & $19.914 \pm 2.904$ \\
$\mathrm{~N}$ & $0.021 \pm 0.001$ & $0.015 \pm 0.001$ & $0.026 \pm 0.001$ & $0.003 \pm 0.001$ & $32.508 \pm 0.006$ \\
$\mathrm{O}$ & $0.005 \pm 0.002$ & $0.011 \pm 0.002$ & $0.005 \pm 0.002$ & $0.002 \pm 0.001$ & $46.889 \pm 1.678$ \\
\hline
\end{tabular}

A, black cumin seeds; B, Iranian Tokhme Sharbati; C, clove buds; D, Shahjeera; E, Abbaszadeh safron; F, organic fenugreek; $\mathrm{G}$, whole black pepper; $\mathrm{H}$, cinnamon; I, Abthul Ahmar (Asario); L, Ajwan seeds; $\mathrm{M}$, whole coriander seeds; N, black sesame seeds; O, Sabja seeds.

Although various classifications for trace elements have been proposed and may be controversial, this paper uses the World Health Organization recommendations which classifies trace elements as: essential trace elements, potentially non-toxic essential elements, and potentially toxic elements [42]. 


\subsubsection{Trace Elements}

Given the increasing consumption of spices and aromatic herbs in the daily diet, it is also interesting to elucidate the content of minerals known for their nutritional roles such as $\mathrm{Cr}, \mathrm{Fe}, \mathrm{Mn}, \mathrm{Cu}, \mathrm{Se}$, and $\mathrm{Zn}$. Several studies on the content of mineral and trace elements in spices and herbs have remarked that they occur in a wide range of concentrations [43-46].

Data on $\mathrm{Cr}$ content in these products are needed. Chromium has been quantified in several foods and beverages, and currently the most comprehensive source are the Danish food composition tables [47]. The content of chromium in foods is relatively low and most foods present a content below $0.1 \mathrm{mg} \mathrm{kg}^{-1}$. Data from the literature indicate that the presence of $\mathrm{Cr}$ in spices and aromatic herbs is higher than other foods and beverages, within a range from 0.01 to $3.0 \mathrm{mg} \mathrm{kg}^{-1}$ [48-51]. In our study, the chromium level found in spices was below $0.3 \mathrm{mg} \mathrm{kg}^{-1}$, except for the high content found in cinnamon $\left(2.35 \mathrm{mg} \mathrm{kg}^{-1}\right)$ and thymol seeds $\left(1.11 \mathrm{mg} \mathrm{kg}^{-1}\right)$.

Selenium content varied from 0.003 to $0.505 \mathrm{mg} \mathrm{kg}^{-1}$; in particular, selenium was reported with values: $0.254,0.287,0.254,0.505$, and $0.485 \mathrm{mg} \mathrm{kg}^{-1}$ for caraways, cress sprouting seeds, thymol seeds, coriander, and black sesame seeds, respectively. Selenium (Se) is an essential trace element involved in the synthesis of various selenium-containing proteins, and also has other relevant biological functions; moreover, it has a fundamental role in the human diet since it may act as a preventive agent against some health conditions [51,52].

\subsubsection{Potentially Non-Toxic Elements}

Eight trace elements that are not normally known for their toxic effects were identified, but it is nonetheless important to monitor them, because if very high concentrations of these metals are ingested, they can lead to physiological disorders [53,54]. Their maximum concentrations were found to be in the decreasing order as follows: $\mathrm{Sr}>\mathrm{Ni}>\mathrm{Sn}>\mathrm{V}>\mathrm{Co}>$ $\mathrm{Sb}>\mathrm{Be}$. All these potentially non-toxic trace elements were contained in variable amounts in the analyzed spices. In particular among these, the major trace elements were: $\mathrm{Sr}$, which ranged from $95.92 \pm 0.20 \mathrm{mg} \mathrm{kg}^{-1}$ (carraway) to $4.30 \pm 0.02 \mathrm{mg} \mathrm{kg}^{-1}$ (fenugreek); $\mathrm{Ni}$, which ranged from $3.05 \pm 0.12 \mathrm{mg} \mathrm{kg}^{-1}$ (black cumin) to $0.42 \pm 0.01 \mathrm{mg} \mathrm{kg}^{-1}$ (chia seeds); $\mathrm{Sn}$, which ranged from $2.39 \pm 0.04 \mathrm{mg} \mathrm{kg}^{-1}$ (coriander) to $0.11 \pm 0.04 \mathrm{mg} \mathrm{kg}^{-1}$ (clove buds); and $\mathrm{Co}$, which ranged from $0.29 \pm 0.00 \mathrm{mg} \mathrm{kg}^{-1}$ (thymol seeds) to $0.02 \pm 0.00 \mathrm{mg} \mathrm{kg}^{-1}$ (black pepper).

\subsubsection{Potentially Toxic Elements}

Concerning $\mathrm{Cd}, \mathrm{Pb}, \mathrm{As}$, and $\mathrm{Hg}$, the following ranges have been observed in the 13 analyzed spices: $\mathrm{Cd}\left(0.003-0.079 \mathrm{mg} \mathrm{kg}^{-1}\right), \mathrm{Pb}\left(0.008-0.544 \mathrm{mg} \mathrm{kg}^{-1}\right)$, As $(0.003-$ $\left.0.339 \mathrm{mg} \mathrm{kg}^{-1}\right)$, and $\mathrm{Hg}\left(0.001-0.010 \mathrm{mg} \mathrm{kg}^{-1}\right)$. The contamination level of the analyzed samples followed the sequence: $\mathrm{Pb}>\mathrm{As}>\mathrm{Cd}>\mathrm{Hg}$.

Heavy metals should be closely monitored, considering that the absorption and bioaccumulation of those compounds, with reference to their toxic and mutagenic effects, have a negative effect on consumers' health $[8,44,45,55]$.

According to the European Commission Regulation (EC) No. 1881/2006 and its amendments, Regulation (EU) No. 1317/2021 and Regulation (EU) No. 1323/2021, the maximum levels in fresh herbs for lead and cadmium have been set at $0.1 \mathrm{mg} \mathrm{kg}^{-1}$ and $0.2 \mathrm{mg} \mathrm{kg}^{-1}$, respectively. However, for many elements, there is a lack of shared worldwide regulation, and reference could be made to the values reported by WHO and EFSA, namely 5.0 and $0.2 \mathrm{mg} \mathrm{kg}^{-1}$ for As and $\mathrm{Hg}$, respectively [28-36,56,57].

Concerning the content of potentially toxic minerals $(\mathrm{Cd}, \mathrm{Pb}, \mathrm{As}$, and $\mathrm{Hg})$, the results showed that all the spices analyzed did not present contamination concerns for cadmium, arsenic, and mercury, as the contents of these three metals were always lower than the permitted limit values established by the European Commission regulation and WHO. On the other hand, for $\mathrm{Pb}$ content, two samples showed two warning values with respect to the established permitted limit of $0.1 \mathrm{mg} \mathrm{kg}^{-1}$. Ajwan seeds from India (sample L) showed 
a lead content $\left(0.544 \mathrm{mg} \mathrm{kg}^{-1}\right)$ five times higher than the permitted limit $(p<0.05)$ while the lead content found in Abbaszadeh saffron from Iran ( $0.096 \mathrm{mg} \mathrm{kg}^{-1}$, sample E) was close to the accepted limit $(p>0.05)$, indicating that for these samples, there was a possible threat to health.

Several studies have reported a potential threat to the nervous system from aluminum $[58,59]$. The content of aluminum ranges from $7.112 \mathrm{mg} \mathrm{kg}^{-1}$ in fenugreek to $46.889 \mathrm{mg} \mathrm{kg}^{-1}$ in chia seeds, with the exception of carraway, saffron, and thymol seeds, in which the reported values were $87.507 \mathrm{mg} \mathrm{kg}^{-1}, 145.216 \mathrm{mg} \mathrm{kg}^{-1}$, and $930.198 \mathrm{mg} \mathrm{kg}^{-1}$, respectively, showing a high capacity to accumulate aluminum.

Lopez et al. [58] showed data on the levels of $\mathrm{Al}$ in 72 dried samples of 17 different spices and aromatic herbs, and aluminum levels ranged from 3.74 to $56.50 \mathrm{mg} \mathrm{kg}^{-1}$. Bratakos et al. found $\mathrm{Al}$ values in spices with a mean value of $157 \mathrm{mg} \mathrm{kg}^{-1}$ [58]. For foods from plant origins, high aluminum concentration could be related to its high content in the soil where the plants are grown, or to the fact that plants grow in acid soils, because its availability depends on soil $\mathrm{pH}[59,60]$.

\section{Conclusions}

This study aims at monitoring the levels of both microbial contamination and trace elements in some spices and aromatic herbs commonly used in the Mediterranean diet. The data indicate that black cumin and Iranian chia seeds presented contamination (total coliform bacteria and Enterobacteriaceae). The concentration of trace elements was variable and related to each spice. Concerning the contents of potentially toxic heavy metals $(\mathrm{Cd}$, $\mathrm{Pb}, \mathrm{As}$, and $\mathrm{Hg}$ ), they were within the above-mentioned limits, although $\mathrm{Pb}$ presented a higher value in two cases.

It should be considered as a final remark that all spices and herbs are susceptible to environmental (e.g., microbial and heavy metal) contamination. Microbial contamination could be prevented by adopting good standards of practice during growing, harvesting, and processing. Environmental contamination with heavy metals should be avoided and monitored to minimize contamination levels. For this reason, spices and aromatic herbs must be strictly monitored for the aspects concerning their safety in order to prevent foodborne illness due to contamination.

Author Contributions: Conceptualization, N.C., A.S., and T.G.; methodology, N.C., T.G., R.V., and A.S.; investigation, A.M., V.N., F.G., and R.T.; writing-original draft preparation, T.G., A.D., N.C., and A.S.; writing-review and editing, T.G., N.C., and A.S.; visualization, R.V., A.D., and M.L.; project administration, N.C. and A.S.; funding acquisition, N.C. and A.S.; supervision, N.C., A.S., and T.G. All authors have read and agreed to the published version of the manuscript.

Funding: This research received no external funding.

Data Availability Statement: Data is contained within the article.

Conflicts of Interest: The authors declare no conflict of interest.

\section{References}

1. Metro, D.; Tardugno, R.; Papa, M.; Bisignano, C.; Manasseri, L.; Calabrese, G.; Gervasi, T.; Dugo, G.; Cicero, N. Adherence to the Mediterranean diet in a Sicilian student population. Nat. Prod. Res. 2018, 32, 1775-1781. [CrossRef]

2. $\quad$ Elviss, N.C.; Little, C.L.; Hucklesby, L.; Sagoo, S.; Surman-Lee, S.; de Pinna, E.; Threlfall, E.J. Microbiological study of fresh herbs from retail premises uncovers an international outbreak of salmonellosis. Int. J. Food Microbiol. 2009, 134, 83-88. [CrossRef] [PubMed]

3. Elshafie, A.E.; Al-Rashdi, T.A.; Al-Bahry, S.N.; Bakheit, C.S. Fungi and aflatoxins associated with spices in the sultanate of Oman. Mycopathologia 2002, 1, 155-160. [CrossRef]

4. Bua, G.D.; Annuario, G.; Albergamo, A.; Cicero, N.; Dugo, G. Heavy metals in aromatic spices by inductively coupled plasmamass Spectrometry. Food Addit. Contam. Part B 2016, 9, 210-216. [CrossRef]

5. Salvo, A.; La Torre, G.L.; Mangano, V.; Casale, K.E.; Bartolomeo, G.; Santini, A.; Granata, T.; Dugo, G. Toxic inorganic pollutants in foods from agricultural producing areas of Southern Italy: Level and risk assessment. Ecotoxicol. Environ. Saf. 2018, 148, 114-124. [CrossRef] 
6. Pecoraro, R.; Marino, F.; Salvaggio, A.; Capparucci, F.; Caro, G.D.; Iaria, C.; Salvo, A.; Rotondo, A.; Tibullo, D.; Guerriero, G.; et al. Evaluation of chronic nanosilver toxicity to adult zebrafish. Front. Physiol. 2017, 8, 1011. [CrossRef]

7. European Commission. REGULATION (EC) No 1334/2008 of the European Parliament and of the Council of 16 December 2008 on flavourings and certain food ingredients with flavouring properties for use in and on foods and amending Council Regulation (EEC) No 1601/91, Regulations (EC) No 2232/96 and (EC) No 110/2008 and Directive 2000/13/EC. Off. J. Eur. Union 2008, L 354/34. Available online: https:/ / eur-lex.europa.eu/legal-content/en/TXT/?uri=CELEX\%3A32008R1334 (accessed on 21 December 2021).

8. World Health Organization. Guidelines for Assessing Quality of Herbal Medicines with Reference to Contaminants and Residues; World Health Organization: Geneva, Switzerland, 2007.

9. Gan, Y.; Wang, L.; Yang, G.; Dai, J.; Wang, R.; Wang, W. Multiple factors impact the contents of heavy metals in vegetables in high natural background area of China. Chemosphere 2017, 184, 1388-1395. [CrossRef]

10. Balali-Mood, M.; Naseri, K.; Tahergorabi, Z.; Khazdair, M.R.; Sadeghi, M. Toxic Mechanisms of Five Heavy Metals: Mercury, Lead, Chromium, Cadmium, and Arsenic. Front. Pharmacol. 2021, 12, 643972. [CrossRef]

11. Jabeen, S.; Shah, M.; Khan, S.; Hayat, M.Q. Determination of major and trace elements in ten important folk therapeutic plants Pakistan. J. Med. Plants Res. 2010, 4, 559-566.

12. Wafaa Muaad, M.; Khalil Abdul-Rezzak, R. Investigate the presence of some heavy metals and microbes in spices. Samarra J. Pure Appl. Sci. 2021, 3, 41-48. [CrossRef]

13. Al-Dalain, S.Y.; Haddad, M.A.; Al-Hawadi, J.S.; Freihat, A.; Arabiat, S.; Al-Qudah, M.M.; Ateyyat, M.A. Determination of heavy metals and bacterial count of some frequently consumed spices in Jordan. Syst. Rev. Pharmacol. 2021, 12, 319-325.

14. Mikušová, P.; Šrobárová, A.; Sulyok, M.; Santini, A. Fusarium fungi and associated metabolites presence on grapes from Slovakia. Mycotoxin Res. 2013, 29, 97-102. [CrossRef] [PubMed]

15. Nazhand, A.; Durazzo, A.; Lucarini, M.; Souto, E.B.; Santini, A. Characteristics, Occurrence, Detection and Detoxification of Aflatoxins in Foods and Feeds. Foods 2020, 9, 644. [CrossRef]

16. Mikusova, P.; Ritieni, A.; Santini, A.; Juhasova, G.; Srobarova, A. Contamination by moulds of grape berries in Slovakia. Food Addit. Contam. Part A Chem. Anal. Control. Expo. Risk Assess. 2010, 27, 738-747. [CrossRef] [PubMed]

17. Santini, A.; Meca, G.; Ritieni, A. Fusaproliferin, Beauvericin, and Enniatins: Occurrence in food-A review. World Mycotoxin J. 2012, 5, 71-81. [CrossRef]

18. Zhao, X.; Jin, X.; Lin, Z.; Guo, Q.; Liu, B.; Yuan, Y.; Yue, T.; Zhao, X. Simultaneous rapid detection of aflatoxin B1 and ochratoxin a in spices using lateral flow immuno-chromatographic assay. Foods 2021, 10, 2738. [CrossRef]

19. Sospedra, I.; Soriano, J.M.; Mañes, J. Assessment of the microbiological safety of dried spices and herbs commercialized in Spain. Plant Food Hum. Nutr. 2010, 65, 364-368. [CrossRef]

20. Vitullo, M.; Ripabelli, G.; Fanelli, I.; Tamburro, M.; Delfin, S.; Sammarco, M.L. Microbiological and toxicological quality of dried herbs. Lett. Appl. Microbiol. 2011, 52, 573-580. [CrossRef]

21. European Commission. Securing the Spices and Herbs Commodity Chains in Europe against Deliberate, Accidental or Natural Biological and Chemical Contamination of Haripur Basin; SPICED Project I FP7 I CORDIS; European Commission: Bruxelles, Belgium, 2013; Project Coordinated by Germany. Available online: https:/ / cordis.europa.eu/project/id/312631 (accessed on 3 February 2022).

22. What Is the Demand for Spices and Herbs on the European Market? Available online: https://www.cbi.eu/market-information/ spices-herbs/what-demand (accessed on 3 February 2022).

23. Śmiechowska, M.; Newerli-Guz, J.; Skotnicka, M. Spices and seasoning mixes in European Union-innovations and ensuring safety. Foods 2021, 10, 2289. [CrossRef]

24. Sagoo, S.K.; Little, C.L.; Greenwood, M.; Mithani, V.; Grant, K.A.; Mc Lauchlin, J.; De Pinna, E.; Threlfall, E.J. Assessment of the microbiological safety of dried spices and herbs from production and retail premises in the United Kingdom. Food Microbiol. 2009, 26,39-43. [CrossRef]

25. Witkowska, A.; Hickey, D.; Alonso-Gomez, M.; Wilkinson, M. The microbiological quality of commercial herb and spice preparations used in the formulation of a chicken supreme ready meal and microbial survival following a simulated industrial heating process. Food Control. 2011, 22, 616-625. [CrossRef]

26. Panebianco, F.; Nava, V.; Giarratana, F.; Gervasi, T.; Cicero, N. Assessment of heavy- and semi-metals contamination in edible seaweed and dried fish sold in ethnic food stores on the Italian market. J. Food Compos. Anal. 2021, 104, 104150. [CrossRef]

27. Salvo, A.; La Torre, G.; Rotondo, A.; Cicero, N.; Gargano, R.; Mangano, V.; Casale, E.; Dugo, G. Multiple analytical approaches for the organic and inorganic characterization of Origanum vulgare L. samples. Nat. Prod. Res. 2019, 33, 2815-2822. [CrossRef] [PubMed]

28. Commission Regulation (EC) No 1881/2006 of 19 December 2006 Setting Maximum Levels for Certain Contaminants in Foodstuffs. Available online: https:/ / eur-lex.europa.eu/LexUriServ/LexUriServ.do?uri=OJ:L:2006:364:0005:0024:EN:PDF (accessed on 31 January 2022).

29. Commission Regulation (EU) 2021/1323 of 10 August 2021 Amending Regulation (EC) No 1881/2006 as Regards Maximum Levels of Cadmium in Certain Foodstuffs. Available online: https:/ / eur-lex.europa.eu/eli/reg/2021/1323/oj (accessed on 31 January 2022).

30. Commission Regulation (EU) 2021/1317 of 9 August 2021 Amending Regulation (EC) No 1881/2006 as Regards Maximum Levels of Lead in Certain Foodstuffs. Available online: https:/ / eur-lex.europa.eu/eli/reg/2021/1317/oj (accessed on 31 January 2022). 
31. EFSA (European Food Safety Authority). Opinion of the scientific panel on dietetic products, nutrition and allergies on a request from the commission related to the tolerable upper intake level of sodium. EFSA J. 2005, 209, 1-26.

32. EFSA (European Food Safety Authority). Scientific opinion on arsenic in food. EFSA J. 2009, 7, 1-199.

33. EFSA (European Food Safety Authority). Scientific opinion on lead in food. EFSA J. 2010, 8, 1-151.

34. EFSA (European Food Safety Authority). Cadmium dietary exposure in the European population. EFSA J. 2012, $10,1-37$.

35. EFSA (European Food Safety Authority). Scientific opinion on the risk for public health related to the presence of mercury and methyl-mercury in food. EFSA J. 2012, 10, 1-241.

36. European Communities Commission. Commission Directive 2008/100/EC of 28 October 2008 Amending Council Directive 90/496. EEC on Nutrition Labelling for Foodstuffs as Regards Recommended Daily Allowances, Energy Conversion Factors and Definitions; European Communities Commission: 2008. Available online: https:/ / eur-lex.europa.eu/LexUriServ/LexUriServ.do? uri=OJ:L:2008:285:0009:0012:EN:PDF (accessed on 22 November 2021).

37. Hammer, O.; Harper, D.A.T.; Ryan, P.D. PAST: Paleontological statistics software package for education and data analysis. Palaeontol. Electron. 2001, 4, 1-9.

38. European Commission. Commission Regulation (EC) No 2073/2005 of 15 November 2005 on microbiological criteria for foodstuffs. Off. J. Eur. Union 2005, 50, 1-26.

39. European Commission. Commission Regulation (EC) No 1441/2007 of 5 December 2007 amending Regulation (EC) No 2073/2005 on microbiological criteria for foodstuffs. Off. J. Eur. Union 2007, 322, 12-29.

40. Halkman, A.K. Indicator Organisms. In Encyclopedia of Food Microbiology, 2nd ed.; Carl, A.B., Mary, L.T., Eds.; Academic Press: Cambridge, MA, USA, 2014; pp. 358-363, ISBN 9780123847331.

41. EURACHEM. Guide. S L R Ellison and A. Williams (Eds). Eurachem/CITAC Guide: Quantifying Uncertainty in Analytical Measurement, Third edition. 2012. ISBN 978-0-948926-30-3. Available online: https://www.eurachem.org/ (accessed on 21 December 2021).

42. WHO (World Health Organization). Trace Elements in Human Nutrition and Health. Geneva, Switzerland. 1996. Available online: https:/ / scholar.googleusercontent.com/scholar?q=cache:1ZTWbPtjCfYJ:scholar.google.com/\&hl=en\&as_sdt=0,5 (accessed on 22 November 2021).

43. Tokalığlu, Ş. Determination of trace elements in commonly consumed medicinal herbs by ICP-MS and multivariate analysis. Food Chem. 2012, 134, 2504-2508. [CrossRef] [PubMed]

44. Ibrahim, G.; Hassan, L.; Baban, S.; Fadhil, S. Effect of heavy metal content of some common spices available in local markets in Erbil City on human Consumption. Raf. J. Sci. 2012, 23, 106-114. [CrossRef]

45. Doabi, S.; Karami, M.; Afyuni, M.; Yeganeh, M. Pollution and health risk assessment of heavy metals in agricultural soil at-mospheric dust and major food crops in Kermanshah province, Iran. Ecotox. Environ. Saf. 2018, 163, 153-164. [CrossRef]

46. Nan, C.; Changhui, Z.; Tiehua, Z. Selenium transformation and selenium-rich foods. Food Biosci. 2021, 40, 100875. [CrossRef]

47. Food Data (frida.fooddata.dk), Version 4; National Food Institute, Technical University of Denmark: Lyngby, Denmark, 2019. Available online: https:/ / frida.fooddata.dk/?lang=en (accessed on 22 November 2021).

48. Castro, A. Chromium in a series of Portuguese plants used in the herbal treatment of diabetes. Biol. Trace Elem. Res. 1998, 62, 101-106. [CrossRef] [PubMed]

49. Abdul Latif, A.; Bilal, M.; Asghar, W.; Azeem, M.; Muhammad, I.; Ahmad, M.I.; Abbas, A.; Ahmad, M.Z.; Toufeeq Shahzad, T. Heavy metal accumulation in vegetables and assessment of their potential health risk. J. Environ. Anal. Chem. 2018, 5, 1000234. [CrossRef]

50. Tefera, M.; Teklewold, A. Health risk assessment of heavy metals in selected Ethiopian spices. Heliyon 2021, 7, e07048. [CrossRef]

51. Garcia, E.; Cabrera, C.; Lorenzo, M.L.; López, M.C. Chromium levels in spices and aromatic herbs. Sci. Total Environ. 2000, 247, 51-56. [CrossRef]

52. Chen, J.; Zhang, Y.; Lv, Y.; Tian, M.; You, J.; Chen, F.; Zhang, S.; Guan, W. Effects of Selenomethionine on Cell Viability, Selenoprotein Expression and Antioxidant Function in Porcine Mammary Epithelial Cells. Front. Nutr. 2021, $26,665855$. [CrossRef]

53. Khan, N.; Jeong, I.S.; Hwang, I.M.; Kim, J.S.; Choi, S.H.; Nho, E.Y.; Choi, J.Y.; Kwak, B.-M.; Ahn, J.K.; Yoo, T.; et al. Method validation for simultaneous determination of chromium, molybdenum and selenium in infant formulas by ICP-OES and ICP-MS Food Chem. 2013, 141, 3566-3570. [CrossRef] [PubMed]

54. Llorent-Martínez, E.J.; De Córdova, M.L.F.; Ruiz-Medina, A.; Ortega-Barrales, P. Analysis of 20 trace and minor elements in soy and dairy yogurts by ICPMS. Microchem. J. 2012, 102, 23-27. [CrossRef]

55. Briffa, J.; Sinagra, E.; Blundell, R. Heavy metal pollution in the environment and their toxicological effects on humans. Heliyon 2020, 6, e04691. [CrossRef] [PubMed]

56. Kowalska, G. The safety assessment of toxic metals in commonly used herbs, spices, tea, and coffee in Poland. Int. J. Environ. Res. Public Health 2021, 18, 5779. [CrossRef]

57. Cilak, G.O.; Mujdeci, G.N.; Kabak, B. Legislation on aromatic herbs in food. In Aromatic Herbs in Food; Charis, M.G., Ed.; Academic Press: Cambridge, MA, USA, 2021; pp. 405-438.

58. López, F.F.; Cabrera, C.; Lorenzo, M.L.; López, M.C. Aluminium levels in spices and herbs. Sci. Total Environ. 2000, $257,191-197$. [CrossRef] 
59. Bojórquez-Quintal, E.; Escalante-Magaña, C.; Echevarría-Machado, I.; Martínez-Estévez, M. Aluminum, a Friend or Foe of Higher Plants in Acid Soils. Front. Plant. Sci. 2017, 8, 1767. [CrossRef]

60. Bratakos, S.M.; Lazou, A.E.; Bratakos, M.S.; Lazos, E.S. Aluminium in food and daily dietary intake estimate in Greece. Food Addit. Contam. Part B Surveill. 2012, 5, 33-44. [CrossRef] 